\title{
Período de descanso dos suínos no frigorífico e seu impacto na perda de peso corporal e em características do estômago
}

\author{
Resting time of pigs at the processing plant: impact on body weight losses and stomach characteristics
}

\author{
Osmar Antonio Dalla Costa ${ }^{1,2^{*}}$ Arlei Coldebella1 ${ }^{1}$ Mateus José Rodrigues Paranhos da Costa ${ }^{3}$ \\ Luigi Faucitano $^{4}$ José Vicete Peloso $^{5}$ Jorge Victor Ludke ${ }^{1}$ Gerson Neudí Scheuermann ${ }^{1}$
}

\section{RESUMO}

O manejo pré-abate dos suínos, quando realizado de maneira inadequada, pode proporcionar perdas qualitativas e quantitativas. $O$ objetivo deste trabalho foi avaliar o efeito do período de descanso dos suínos no frigorífico (PDF) (3, 5, 7 e 9 horas) e da posição do box na carroceria do caminhão (PBO) sobre a perda de peso corporal (PPC), o peso do estômago cheio (PEC) e vazio (PEV), o peso do conteúdo estomacal (PCE) e o escore de lesão na mucosa esofágicagástrica (ELG). Foram utilizadas 192 fêmeas, com peso vivo médio de $130,0 \pm 9,9 \mathrm{~kg}$. Para a análise estatística, considerouse no modelo os efeitos de bloco $(B L)$, relativo à estação do ano e à granja avaliada o PDF, $P B O$ e a interação entre $P D F \times B L$. Não houve efeito significativo do PDF nas variáveis estudadas. Verificou-se efeito significativo da PBO somente sobre a perda de peso corporal dos suínos, sendo que animais transportados na parte da frente perderam mais peso em relação aos transportados nos boxes do meio e de trás. Observou-se que somente $11 \%$ dos suínos apresentaram ELG característico de úlcera gástrica e que uma elevada porcentagem de suínos (41,9\%) apresentou ELG grau 1, caracterizando paraqueratose. Conclui-se que suínos transportados em boxes localizados na parte frontal da carroceria do caminhão apresentam maior perda de peso corporal do que os demais e que a variação de 3 a 9 horas no período de descanso no frigorífico não afeta a perda de peso corporal nem as características do estômago dos animais.

Palavras-chave: suínos, manejo pré-abate, transporte, período de descanso, peso corporal, úlcera gástrica.

\section{ABSTRACT}

Pre-slaughter events might result in qualitative and quantitative losses on pig carcasses in inadequate management conditions. This study was aimed at evaluating the resting time at the processing plant $(P D F=3,5,7$, and 9 hours) and the pen position in the lorry's livestock compartment $(P B O)$ on body weight loss (PPC), weight of stomach (full = PEC and empty $=P E V)$, weight of stomach contents (PCE) and stomach lesion score (ELG) related to gastric ulcer. For this study, 192 females with average body weight of $130.0 \pm 9.9 \mathrm{~kg}$ were used. The following effects were considered in the statistical analysis: block (season of the year), PDF, PBO and the interaction between block and PDF. There was no significant effect of PDF on the evaluated variables. For PBO, a significant effect was observed on body weight loss, with pigs transported in the frontal pens losing more body weight than those transported in middle and back pens. Eleven percent of the animals showed ELG characterizing gastric ulcer, while a high percentage of the pigs (41.9\%) showed ELG grade 1, which characterizes paracheratosis. It is concluded that pigs transported in the frontal pens of the lorry's livestock compartment do have higher body weight losses, and that increasing the resting time at the processing plant from 3 to 9 hours does not impact body weight losses or the incidence of gastric ulcer.

Key words: pigs, pre-slaughter events, transport, resting time, body weight, gastric ulcer.

\section{INTRODUÇÃO}

O manejo pré-abate é um processo complexo, que inclui uma série de atividades envolvendo a preparação e o manejo dos animais para o transporte e abate, quais sejam: jejum na granja, embarque, transporte, desembarque, alojamento nas baias do frigorífico, período de descanso,

\footnotetext{
${ }^{1}$ Embrapa Suínos e Aves, CP 21, 89700-000, Concórdia, SC, Brasil. E-mail: osmar@cnpsa.embrapa.br. *Autor para correspondência. ${ }^{2}$ Programa de Pós-graduação em Zootecnia, Faculdade de Ciência Agrárias e Veterinárias (FCAV), Universidade Estadual Paulista (UNESP), Jaboticabal, SP, Brasil. ETCO (Grupo de Estudos e Pesquisa em Etologia e Ecologia Animal).

${ }^{3}$ Departamento de Zootecnia, ETCO, FCAV, UNESP, 14870-000, Jaboticabal, SP, Brasil.

${ }^{4}$ Agriculture and Agri-Food Canada, Dairy and Swine Research and Development Centre, P.O. Box 90, 108 Route East, Lennoxville, Quebec, Canada.

${ }^{5}$ Sadia S. A. Concórdia, SC, Brasil.
} 
atordoamento e abate. Se essas atividades não forem realizadas adequadamente podem ocorrer perdas quantitativas e qualitativas na carne. São muitos os potenciais agentes estressores envolvidos no manejo pré-abate, os quais podem ocasionar alterações fisiológicas e metabólicas decorrentes do desconforto físico e/ou emocional.

O período de descanso no frigorífico é uma importante prática de manejo, que tem sido utilizada para possibilitar a recuperação dos animais do estresse físico e emocional ocorrido no transporte. Além disso, este período auxilia na manutenção da velocidade constante da linha de abate. Para tanto, é usual deixar os animais em repouso nas baias do frigorífico de 1 a 3 horas, tempo necessário para a recuperação das reservas de glicogênio, o que possibilita a produção de carcaças de boa qualidade, baseado em avaliações de $\mathrm{pH}$, cor e perda de água (WARRISS et al., 1998). Contudo, o período de descanso ótimo depende da logística do estabelecimento (distância da granja ao abatedouro, condições de transporte, mistura de lotes, temperatura ambiente) e da intensidade do estresse a que os suínos foram submetidos durante o manejo préabate (GEVERINK et al., 1996; SANTOS et al., 1997; GIESPERT et al., 2000).

Alguns estudos têm avaliado o impacto do período de descanso em variáveis de interesse relacionadas à quantidade e à qualidade da carne suína. Foi observado que períodos curtos de descanso no frigorífico não tiveram efeito no peso (PÉREZ et al., 2002; NANNI COSTA et al., 2002) nem no rendimento da carcaça quente (MURRAY et al., 2001) dos suínos. Porém, suínos que descansaram por longos períodos (24 horas) apresentaram menor peso de carcaça quente em relação a animais que tiveram curtos períodos de descanso ( $\leq 1$ e 3 horas) (WARRISS et al., 1998).

É importante considerar que as transformações bioquímicas do músculo no período post mortem são $\mathrm{pH}$-dependentes, sendo o $\mathrm{pH}$ influenciado por fatores relacionados ao manejo préabate. Por isso, a variável pH é mensurada normalmente nos período de 45 minutos (pHi) e 24 horas ( $\mathrm{pHu}$ ) após o abate, visando à avaliação da qualidade da carne.

O objetivo deste trabalho foi avaliar o efeito da posição dos suínos na carroceria do caminhão durante o transporte da granja até o frigorífico e do período de descanso dos suínos no frigorífico sobre a perda de peso corporal, os pesos do estômago e seu conteúdo e o escore de lesão na mucosa esofágicagástrica (ELG).

\section{MATERIAL E MÉTODOS}

Foram utilizadas 192 fêmeas suínas oriundas de cruzamentos industriais com peso vivo médio de $128,3 \pm 3,6 \mathrm{~kg}$ no inverno e de $131,7 \pm 4,5 \mathrm{~kg}$ no verão e período de alojamento médio de 144 dias para as fases de crescimento e terminação. Os animais foram obtidos de duas granjas (correspondendo aos blocos) que operam em sistema de terminação, sendo uma no verão (fevereiro de 2002) e outra no inverno (julho de 2002). Em cada granja avaliada foram escolhidas aleatoriamente 4 baias/período de descanso (6 animais/ baia), totalizando 16 baias/granja para a realização do experimento, em um total de 96 suínos/granja.

No manejo pré-abate, os suínos receberam tempo de jejum na granja antes do carregamento de 12 horas. No desembarque dos suínos no frigorífico, utilizou-se uma rampa móvel, sendo que os animais receberam períodos de descanso no frigorífico de 3,5, 7 e 9 horas. Os suínos foram pesados uma hora após a última refeição e imediatamente antes do abate para a obtenção da perda de peso corporal (PPC).

$\mathrm{O}$ transporte dos suínos foi realizado em um modelo de carroceria metálica dupla, TRIEL-HT, com capacidade de transporte de 96 animais,_representando uma densidade média de $0,45 \mathrm{~m}^{2}$ animal ${ }^{-1}$ ou $288 \mathrm{~kg} \mathrm{~m}^{-2}$. Esta carroceria estava sobre um chassi Volkswagen trucado modelo $24.220(6 \times 4)$.

No frigorífico, os suínos foram desembarcados com o auxílio de uma plataforma móvel conduzida até as baias de descanso coletivas. No desembarque e deslocamento dos animais até as baias de descanso, estes foram misturados aleatoriamente dentro de cada grupo de período de descanso. A insensibilização (ou eletro-narcose) foi aplicada automaticamente, transferindo alta voltagem $(700 \mathrm{~V}) \mathrm{e}$ amperagem acima de 1,25 Amps (Valhalla, Stork RMS b.v., Lichtenvoorde, Holanda). Após a insensibilização, os animais foram imediatamente sangrados na posição horizontal e suspensos ao fim da mesa de sangria na nórea contínua da linha de abate. Durante o processo de evisceração dos animais em avaliação, coletou-se o estômago, o qual foi pesado cheio (PEC) e vazio (após a retirada do conteúdo estomacal e da limpeza com água corrente) (PEV), pesando-se também o conteúdo estomacal (PCE), classificado em quatro categorias, conforme CHEVILLON (1994). Após a limpeza dos estômagos, os mesmos foram examinados macroscopicamente para avaliação do escore de lesão na mucosa esofágica-gástrica (ELG), seguindo classificação proposta por MORÉS et al. (2000).

As carcaças dos suínos permaneceram em câmara fria, submetidas a temperaturas variando entre 
$1^{\circ} \mathrm{C}$ e $4^{\circ} \mathrm{C}$ por 24 horas. As medidas do $\mathrm{pH}$ foram realizadas na meia carcaça esquerda nos músculos Longissimus dorsi (LD) e Semimembranosus (SM), 45 minutos $\left(\mathrm{pH}_{1}\right)$ e 24 horas após o abate $\left(\mathrm{pH}_{\mathrm{U}}\right)$. Na avaliação do $\mathrm{pH}$, foi utilizado o $\mathrm{pHmetro}$ portátil da marca Mettler Toledo (MP 120 pH Meter, Suíça), com eletrodo DXK-S7/25 protegido para inserção no músculo.

As análises de variância foram realizadas utilizando-se o método de quadrados mínimos, aplicando-se o procedimento GLM do programa SAS (2001), conforme o seguinte modelo matemático:

$\mathrm{Y}_{\mathrm{ijklmn}}=\mu+\mathrm{BL}_{\mathrm{i}}+\mathrm{PDF}_{\mathrm{j}}+(\mathrm{BL} \times \mathrm{PDF})_{\mathrm{ij}}+\mathrm{PBO}_{\mathrm{k}}+\mathrm{PPI}_{1}+$ $\mathrm{PLA}_{\mathrm{m}}+\mathrm{COV}+\mathrm{e}_{\mathrm{ijklmn}}$

sendo,

$\mathrm{Y}_{\mathrm{ijklmn}}=$ PPC, \%PPC, PEC, PEV PCE;

$\mu=$ média geral; $\mathrm{BL}_{\mathrm{i}}=$ bloco (estação do ano, $\mathrm{i}=1$ inverno e 2 verão);

$\mathrm{PDF}_{\mathrm{j}}=$ período de descanso dos suínos no frigorífico antes do abate $\mathrm{j}=1$ (descanso de 3 horas), 2 (descanso de 5 horas); 3 (descanso de 7 horas) e 4 (descanso de 9 horas);

$\mathrm{PBO}_{\mathrm{k}}=$ posição do animal na carroceria, $\mathrm{k}=1$ (frente), 2 (meio) e 3 (atrás);

$\mathrm{PPI}_{1}=$ piso da carroceria, $1=1$ (inferior) e 2 (superior); $\mathrm{PLA}_{\mathrm{m}}=$ lado da carroceria, $\mathrm{m}=1$ (direto) e 2 (esquerdo); $(\mathrm{BL} \times \mathrm{PDF})_{\mathrm{ij}}=$ interação entre bloco e período de descanso dos suínos no frigorífico;

$\mathrm{COV}=$ covariável de interesse para estudo da variável dependente (perda de peso durante o manejo pré-abate $(\mathrm{kg})$ ajustado pelo peso dos suínos na granja antes do carregamento);

$\mathrm{e}_{\mathrm{ijk} k \mathrm{mn}}=$ erro aleatório.

O ELG foi analisado pelo Teste de QuiQuadrado.

\section{RESULTADOS E DISCUSSÃO}

Não se observou efeito significativo do período de descanso dos suínos no frigorífico sobre a $\mathrm{PPC}(\mathrm{P}=0,787)$ e a $\% \mathrm{PPC}(\mathrm{P}=0,811)$, como caracterizado na figura 1. Esses resultados estão de acordo com os obtidos por MURRAY et al. (2001), que verificaram não haver diferença entre os períodos de descanso de 0 a 1; 2 a 3 e 4 a 5 horas quanto ao efeito sobre o rendimento de carcaça de suínos. De forma semelhante, PÉREZ et al. (2002) e NANNI COSTA et al. (2002) também não encontraram efeito do período de descanso $(0,3$ e 9 horas e 2 e 24 horas, respectivamente) dos suínos no frigorífico sobre o peso da carcaça quente dos animais. Já para períodos de descanso maiores 24 horas,
WARRISS et al. (1998) verificaram redução significativa do peso de carcaça em relação aos animais que descansaram somente uma ou três horas.

Possivelmente, o fato de não se ter observado efeito do período de descanso dos suínos no frigorífico deve-se à duração do jejum na granja (12 horas), à distância da granja e do frigorífico $(33 \mathrm{~km})$, à duração do transporte (52min) e aos períodos de descanso no frigorífico ( $3,5,7$ ou 9 horas), o que totaliza aproximadamente $16 \mathrm{a} 22$ horas.

A localização do box (frente, meio e atrás) dentro da carroceria influenciou significativamente $(\mathrm{P}=0,001)$ na PPC e na \%PPC. Suínos que foram transportados no box da frente perderam mais peso do que os animais transportados na parte do meio ou de trás, sendo que estes não diferiram significativamente entre si $(\mathrm{P}<0,050)$. Suínos transportados nos boxes da frente estão mais próximos da cabine do motorista. Nessa região, o estresse térmico é maior devido à proximidade da transmissão mecânica do veículo, que pode ter contribuído significativamente na perda de peso dos animais. Entretanto, não foi observado efeito significativo dos fatores piso (inferior e superior) e lado (direito e esquerdo), $\mathrm{PPC}(\mathrm{P}=0,106$ e $\mathrm{P}=0,243)$ e na $\% \mathrm{PPC}(\mathrm{P}=0,103$ e $\mathrm{P}=0,245)$, conforme apresentado na figura 1.

Os dados de PEC, PEV e PCE são apresentados na figura 2 , de acordo com o período de descanso dos suínos no frigorífico e a posição dentro da carroceria do caminhão (box, piso e lado). O período de descanso não apresentou efeito significativo nas variáveis $\mathrm{PEC}(\mathrm{P}=0,792), \operatorname{PEV}(\mathrm{P}=0,094)$ e $\mathrm{PCE}(\mathrm{P}=0,582)$, resultado que está de acordo com MURRAY et al. (2001), que também não encontraram efeito do período de descanso dos suínos no frigorífico ( 0 a 1; 2 a 3 e 4 a 5 horas) sobre PEC, PEV e PCE. Entretanto, os resultados do presente estudo diferem das observações de KOHLER (2001), que, estudando o efeito do período de descanso (3 e 9 horas) dos suínos no frigorífico, encontrou efeito sobre o PCE, sendo que os animais que descansaram por 3 horas tinham o dobro do PCE (437 vs 220g) em comparação aos suínos que descansaram por 9 horas.

Independentemente do período de descanso ao qual os suínos foram submetidos no frigorífico, não foram encontrados animais com PCE maior que $1.100 \mathrm{~g}$, peso que caracterizaria suínos com estômago cheio. Dois animais $(1,07 \%)$ apresentaram PCE entre $800 \mathrm{e}$ $1.100 \mathrm{~g}$, indicativo de suínos com tendência a estômago cheio, os quais eram oriundos do grupo que descansou 3 horas. Dos 187 suínos avaliados no presente estudo, 


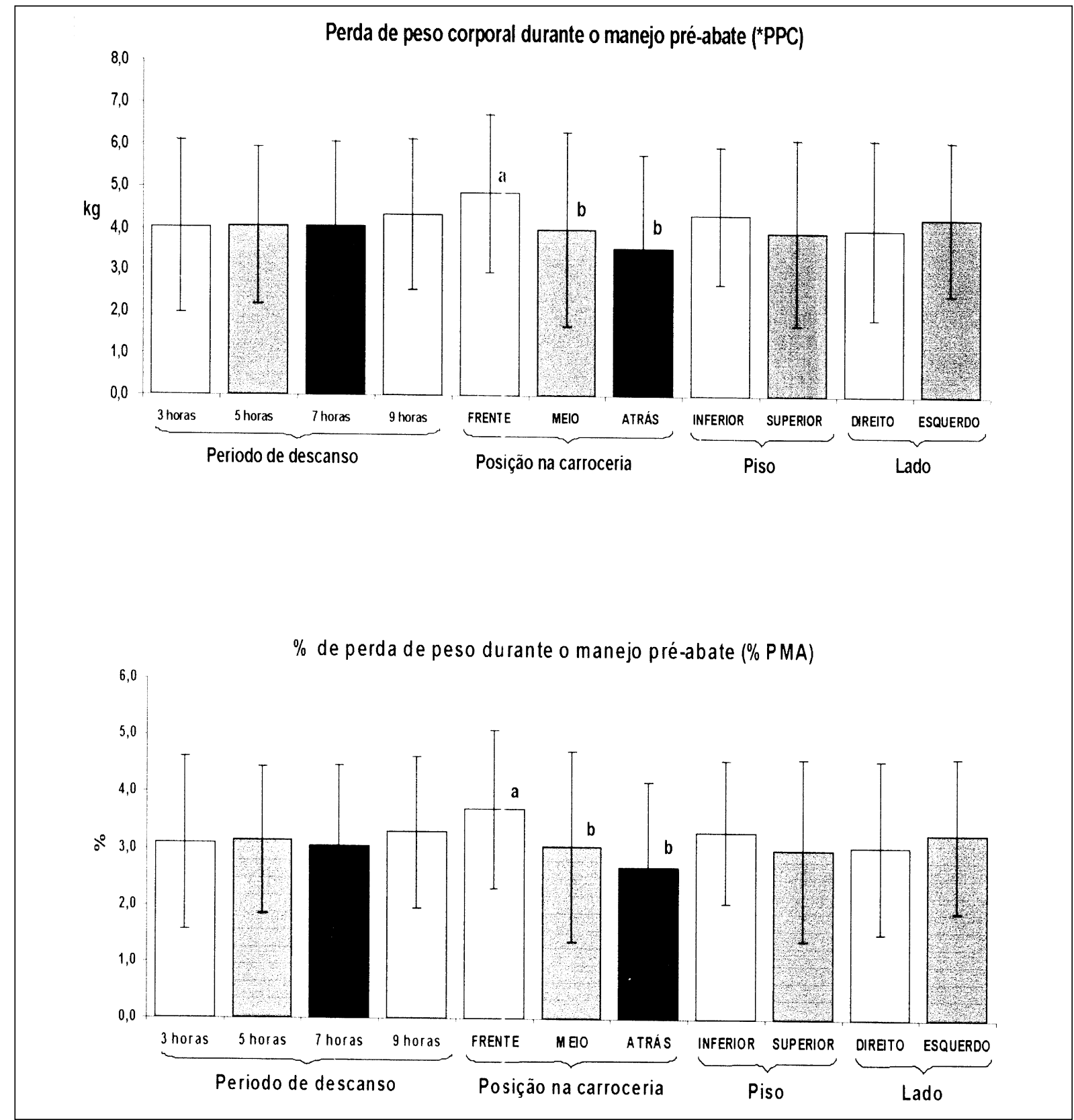

Figura 1 - Médias ajustadas e desvios padrão da perda de peso corporal (PPC) e da porcentagem de PPC (\%PPC) por período de descanso dos suínos no frigorífico e da posição do box dentro da carroceria do caminhão. * Variável ajustada pelo peso do suíno na granja antes do carregamento $(\mathrm{P}=0,0001)$. Médias com letras distintas dentro de cada fator indicam diferença significativa pelo teste $\mathrm{T}(\mathrm{p}<0,05)$.

169 apresentaram PCE menor que 500g, indicativo de suínos com estômagos vazios (CHEVILLON, 1994). Foi verificado incremento do número de suínos com o PCE menor que $500 \mathrm{~g}$ com o aumento do período de descanso no frigorífico de 3, 5, 7 e 9 horas (40, 42, 43 e 44 suínos, respectivamente). Algumas agroindústrias estabelecem o PCE de 200g como meta, pois um PCE maior tende a aumentar a incidência de furos nos intestinos durante o processamento.

Esses resultados estão de acordo com os estudos realizados por LYNCH et al. (2004) em dois frigoríficos, onde foi observado que a maioria dos suínos avaliados (54 a 61\%) apresentava PCE menor que $400 \mathrm{~g}$ e somente 6 a $10 \%$ dos suínos apresentaram 


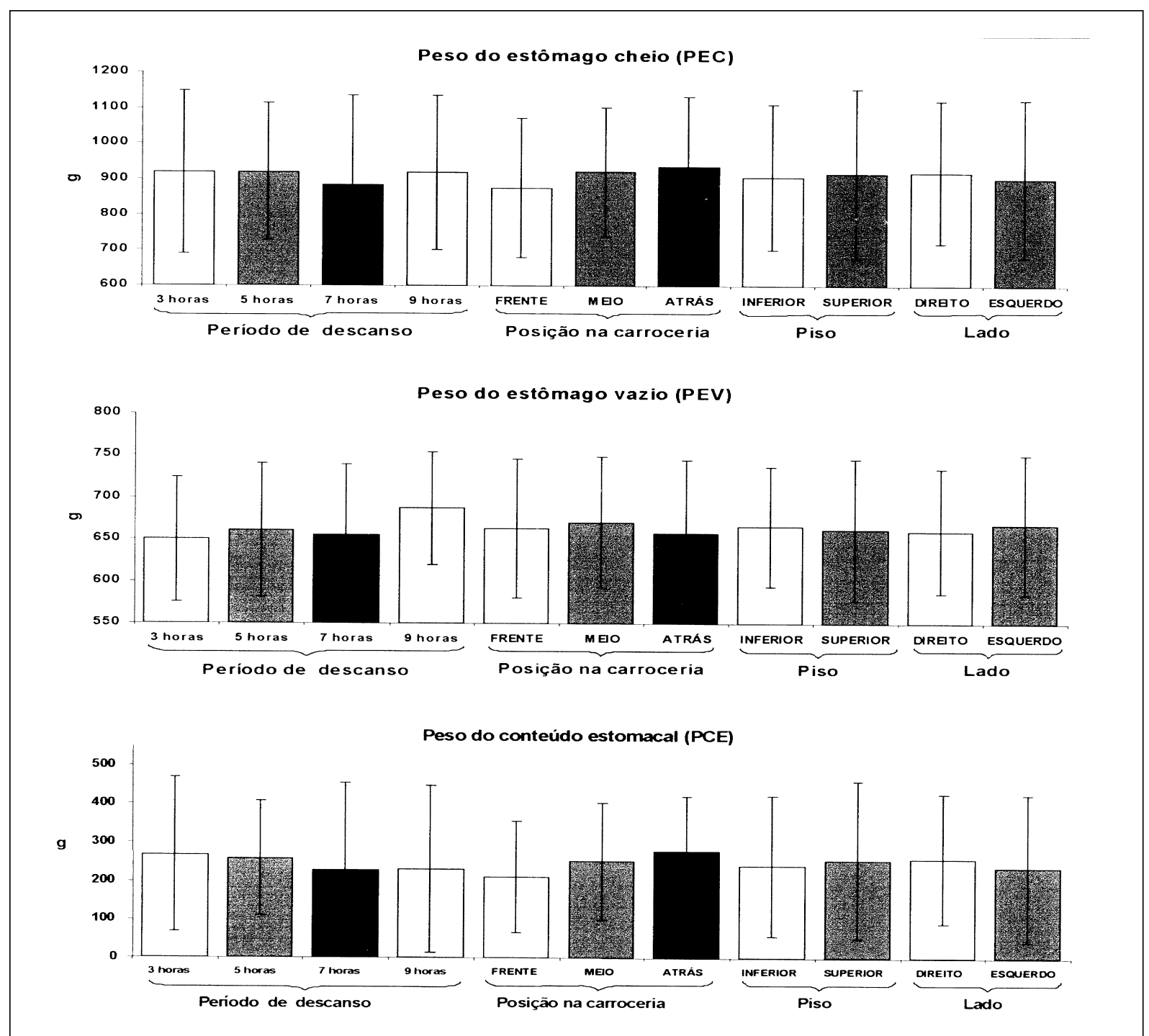

Figura 2 - Médias ajustadas e desvios padrão do peso de estômago cheio (PEC) e vazio (PEV) e conteúdo estomacal (PCE) pelo período de descanso dos suínos no frigorífico e da posição do box dentro da carroceria do caminhão.

PCE maior que $1.000 \mathrm{~g}$. Em estudo relatado por GUISE et al. (1995), avaliando o peso do estômago e de seu conteúdo em 1.242 suínos oriundos de 15 granjas, foram observados valores de CE maiores (540 vs $220 \mathrm{~g}$ ) e PEV semelhantes (680 vs $665 \mathrm{~g}$ ) aos do presente estudo. No mesmo estudo, os autores observaram que $54 \%$ dos estômagos avaliados apresentavam PCE de até $490 \mathrm{~g}$ e somente $12 \%$ tinham PCE maior que $1000 \mathrm{~g}$.

Os três fatores relacionados à carroceria do caminhão que foram avaliados, localização do box, piso e lado, não influenciaram significativamente as variáveis PEC $(\mathrm{P}=0,317 ; \mathrm{P}=0,709$ e $\mathrm{P}=0,709), \mathrm{PEV}(\mathrm{P}=0,638$; $\mathrm{P}=0,743$ e $\mathrm{P}=0,743)$ e $\mathrm{PCE}(\mathrm{P}=0,166 ; \mathrm{P}=0,551$ e $\mathrm{P}=0,551)$, respectivamente.
A ocorrência de úlcera esofágica-gástrica é usual nas diferentes fases da criação dos suínos, sendo sua etiologia complexa e considerada doença multifatorial (MORÉS, 1998). No presente estudo, observou-se que $47,1 \%$ dos animais apresentaram mucosa esofágica-gástrica normal (ELG=0), 41,9\% apresentaram paraqueratose $(\mathrm{ELG}=1), 10,5 \%$ apresentaram $E L G=2$ e apenas $0,5 \%$ evidenciaram ELG de grau 3. Não foi observado efeito significativo $(\mathrm{P}=0,459)$ do período de descanso sobre o ELG (Figura 3). Contudo, BIDNER (1999) verificou que suínos submetidos a jejum de 60 horas apresentaram maior ELG que animais submetidos a jejum de 36 horas. O mesmo autor verificou também que suínos 


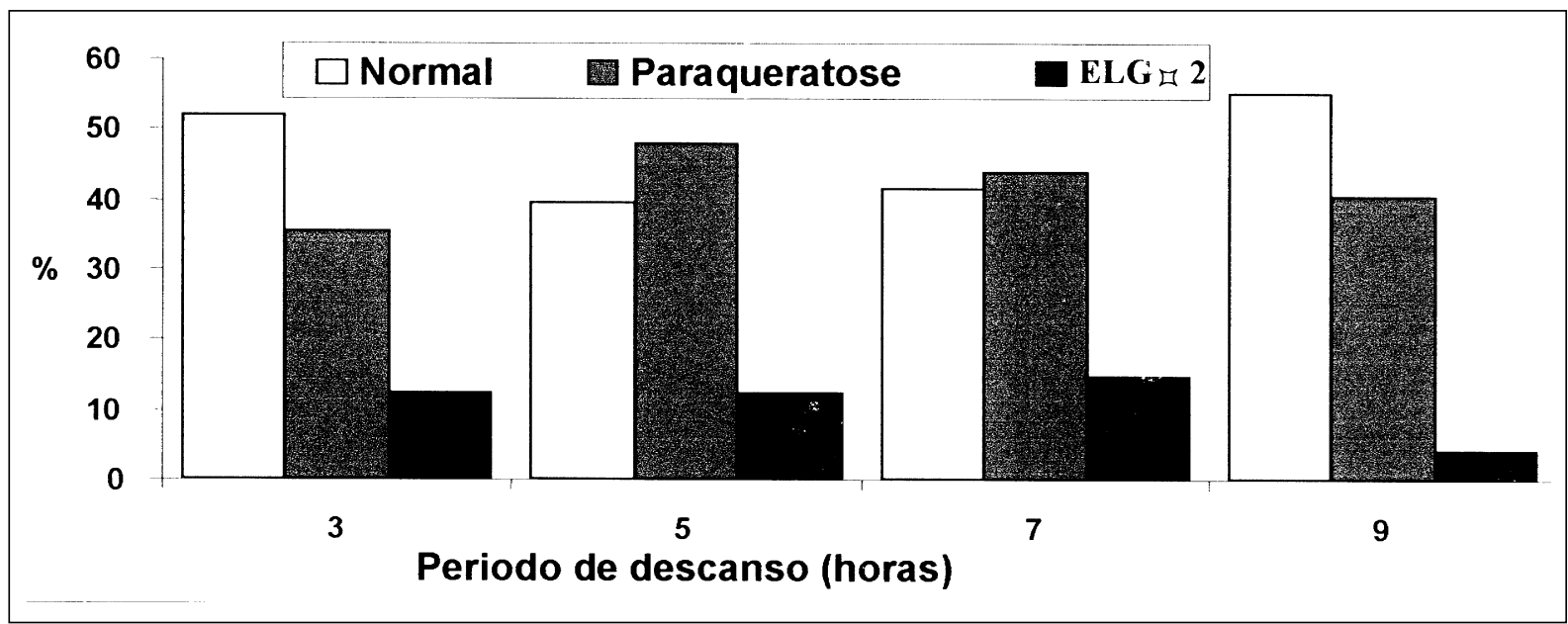

Figura 3 - Média da porcentagem de suínos com estômago normal (sem lesão, ELG=0), com paraqueratose (ELG=1) ou com úlcera esofágica-gástrica (ELG $\geq 2)$, em função período de descanso dos suínos no frigorífico.

submetidos a somente 12 horas de jejum não apresentaram estômago com problemas de paraqueratose ou úlcera, o que está de acordo com os resultados do presente estudo, cujo tempo total de jejum variou de 16 a 22 horas.

$\mathrm{Na}$ figura 4 é apresentada a distribuição das carcaças quanto às faixas de $\mathrm{pH}_{\mathrm{U}}$ observadas nos músculos Longissimus dorsi (LD) e Semimembranosus (SM). As faixas de $\mathrm{pH}_{\mathrm{U}}$ foram divididas em três categorias: baixo $(\leq 5,55)$, normal $(5,55<\mathrm{pHU} \leq 5,90) \mathrm{e}$ elevado $(>5,90)$, considerando os estômagos normais e os com ELG $\leq 2$. Não houve associação significativa entre ELG e a classificação das carcaças quanto ao $\mathrm{pH}_{\mathrm{U}}$ dos músculos $\mathrm{LD}(\mathrm{P}=0,252)$ e $\mathrm{SM}(\mathrm{P}=0,227)$, apesar de observar-se maior incidência de carcaças com $\mathrm{pH}_{\mathrm{U}}$ baixo $(\leq 5,55)$ nos músculos de suínos com estômago normal $(51,2 \%)$ que nos de animais com $E L G \geq 2(35,0 \%)$. Observou-se também que houve baixa porcentagem de suínos com carcaças de $\mathrm{pH}$ elevado, independente do ELG.

\section{CONCLUSÕES}

O presente estudo possibilita concluir que suínos transportados em boxes localizados na parte frontal da carroceria do caminhão apresentam maior perda de peso corporal que os demais e que a variação de 3 a 9 horas no período de descanso no frigorífico não afeta a perda de peso corporal dos animais nem o escore de lesão esofágica-gástrica e o conteúdo estomacal.

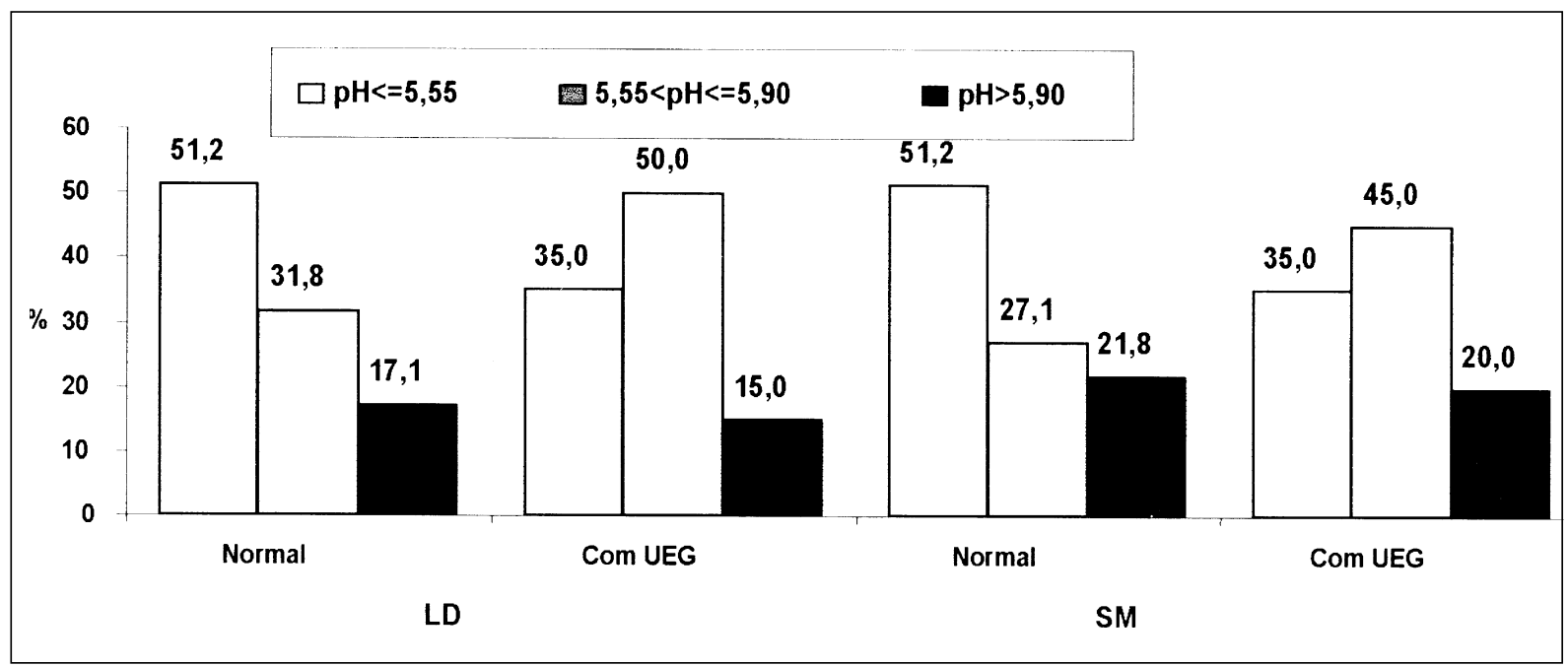

Figura 4 - Distribuição das classes de $\mathrm{pH}_{\mathrm{U}}$ dos músculos Longissimus dorsi (LD) e Semimembranosus (SM) em relação aos estômagos normal (sem lesão) e com úlcera esofágica-gástrica (UEG).

Ciência Rural, v.36, n.5, set-out, 2006. 


\section{REFERÊNCIAS}

BIDNER, E.S. The effects of RN geneotype, feed withdrawal prior to slaughter, lysine-defficient diet, and sodium tripolyphosphate pumping on pork quality and sensory characteristics. 1999. 91f. Dissertação (Mestrado em Zootecncia) - University Illinois.

CHEVILLON, P. Le contrôle des estomacs de porcs á l'abattoir: miroir de la mise à jeun en élevage. Techini-Porc, v.17, p.2330, 1994.

GEVERINK, N.A. et al. Observations on behaviour and skin damage of slaughter pigs and treatment during lairage. Applied Animal Behaviour Science, v.50, p.1-13, 1996.

GIESPERT, M. et al. A survey of pre-slaughter conditions, halothane gene frequency, and carcass and meat quality in five Spanish pig commercial abattoirs. Meat Science, v.55, p.97$106,2000$.

GUISE, H.J. et al. Abattoir observations of the weights of stomachs and their contents in pigs slaughtered at known times after their last feed. British Veterinary Journal, v.151, p.659-670, 1995 .

KOHLER, R.G. Estudo da redução do tempo de permanência na pocilga do frigorífico sobre a qualidade da carne suína. 2001. 77f. Dissertação (Mestre Tecnologia de Alimentos-Setor de Tecnologia) - Universidade Federal do Parará.

LYNCH, P.B. et al. Studies on pre-slaughter handling of pigs and its relationship to meat quality. Acesso em 10 dez. 2004. On line. Disponível na Internet: http:// www.teagasc.ie/research/reports/pigs/4130/eopr-4130.pdf.
MORÉS, N. Influência da granulometria de ingredientes de dietas no desenvolvimento de lesões gástricas em suínos. In: SIMPÓSIO SOBRE GRANULOMETRIA DE INGREDIENTES E RAÇÕES PARA SUÍNOA E AVES, 1998, Concórdia. Anais... Concórdia: EMBRAPA suínos e aves, 1998. p.13-25. (Embrapa suínos e aves. Documentos, 52).

MORÉS, N. et al. Avaliação patológica de suínos no abate manual de identificação. Brasília: Embrapa Comunicação para Transferência de tecnologia, 2000. 40p.

MURRAY, C. et al. Effect of preslaughter overnight feed withdrawal on pig carcass and muscle quality. Canadian Journal of Animal Science, v.81, p.89-97, 2001.

NANNI COSTA, L. et al. Combined effects of pre-slaughter treatments and lairage time on carcass and meat quality in pigs of different halothane genotype. Meat Science, v.61, p.4147, 2002 .

PÉREZ, M.P. et al. Influence of lairage time on some welfare and meat quality parameters in pigs. Veterinany Record, v.33, p.239-250, 2002.

SANTOS, C. et al. Influence of lairage environmental conditions and resting time on meat quality in pigs. Meat Science, v.45, p.253-262, 1997.

SAS INSTITUTE. System for Microsoft Windows: release 8.2. Cary, 2001. 1 CD-ROM.

WARRISS, P.D. et al. Effect of lairage time on levels of stress and meat quality in pigs. Animal Science, v.66, p.255 261, 1998. 